\title{
Self-organizing social hierarchy and villages in a
} challenging society

\author{
Masaru Tsujiguchi and Takashi Odagaki* \\ Department of Physics, Kyushu University, Fukuoka 812-8581, Japan
}

\begin{abstract}
We show by Monte Calro (MC) simulation that the hierarchy and villages emerge simultaneously in a challenging society when the population density exceeds a critical value. Our results indicate that among controlling processes of diffusion and fighting of individuals and relaxation of wealth, the trend of individuals challeninging to stronger neighbors plays the pivotal role in the selforganization of the hierarchy and villages.
\end{abstract}

PACS: 05.65.+b, 05.70.Fh, 64.60.Cn, 68.18.Jk

Keywords: Self-organization; Hierarchy; Phase transition; Social structure

\section{Introduction}

Social structure in various forms exists in the human society and in animals. In the Middle Ages, many villages existed each of which was ruled by a feudal lord and his clan. At present, several nations dominate the world with many followers and some challengers. A key question is how to understand the universal nature in the emergence of these hierarchies which consist of a small number of winners and many losers. It is also an important question to find the mechanism for the simultaneous emergence of the villages and the hierarchy.

Basically, social difference occurs when two moving individuals meet and fight each other where the winner deprives the loser of wealth or power. The winning probability 
of a fight depends on the difference between wealth of two individuals engaging in the fight. Furthermore, the wealth of an individual decays to and the negative wealth (debt) increases to zero when the individual does not fight. Many aspects of the society can be modeled by setting rules to diffusion, fighting and relaxation processes.

In this paper, we consider a challenging,or bellicose society where individuals try to challenge thier neibours if possible. We show by Monte Carlo (MC) simulation that the critical population density for emergence of the hierarchy is much lower than those in the no-preference society[1] and in a timid society[2]. Furthermore, we show that the hierarchy and villages emerge simultaneously in this society; in the no-preference society [1] or in a timid society [2], the hierarchy emerges spontaneously but no villages are observed. Namely, we show that among controlling processes, the trend of individuals challenging to stronger neighbors plays the critical role in the self-organization of the structure.

We organize this paper as follows; in Sec. 2, a challenging society is modelled by setting hostile move of individuals. The results of the MC simulation is presented in Sec. 3 where the density dependence of the order parameter and the profile of winning probability. We also show the formation of villages in the challenging society. Section 4 is devoted to discussion.

\section{A challenging society}

Bonabeau et al. [1] have shown that a hierarchical society can emerge spontaneously from an equal society by a simple algorithm of fighting between individuals who diffuse on a square lattice by a one step simple random walk. Suppose individual $i$ tries to move onto the site occupied by individual $j$ and these two individuals engage in a fighting. The fighting rule is characterised by the winning probability $w_{i j}$ of individual $i$ against individual $j$ which is assumed to be

$$
w_{i j}=\frac{1}{1+\exp \left\{\eta\left(F_{j}-F_{i}\right)\right\}}
$$

where $F_{i}$ is the wealth of individual $i$ and $\eta(>0)$ is a controlling parameter of the model. Therefore, when the difference of the wealths is large, the stronger one wins all the fights, and when $F_{i} \simeq F_{j}$, the winning probability deviates from $1 / 2$ linearly in the 
difference $F_{i}-F_{j}$. The winner occupies the lattice site and increases its wealth by 1 , and the loser moves to the site previously occupied by $i$ and reduces its wealth by 1 .

When individual $i$ is not involved in any fight in one MC time step (MC tries during which all idividuals are accessed once), its wealth is assumed to decay as

$$
F_{i}(t+1)=F_{i}(t)-\mu \tanh \left[F_{i}(t)\right]
$$

where the unit of time is one MC step. When the wealth is large, it decays by a constant amount per one $\mathrm{MC}$ step, $F_{i}(t+1)=F_{i}(t)-\mu$, i.e. a rich person does not waste his/her wealth. When the wealth is small, it decreases at a constant rate, that is $F_{i}(t+1)=(1-\mu) F_{i}(t)$. Here, $\mu$ is another controlling parameter of the model.

The social hierarchy can be characterized by the fact that some people have won and some other people have lost more fights. Suppose individual $i$ won $W_{i}$ times in $X_{i}$ fights for a given time interval. Then the order parameter $\sigma$ can be defined by the mean square deviation of $W_{i} / X_{i}$ from $1 / 2$,

$$
\sigma^{2}=\frac{1}{N} \sum_{i}\left\{\frac{W_{i}}{X_{i}}-\frac{1}{2}\right\}^{2} .
$$

Bonabeau et al showed by MC simulation that the social hierarchy self-organizes at a

critical density as the population density is increased. Note that the relaxation process plays a critical role to have such a transition [3, 4].

In order to study the emergence of social hierarchy and villages in the society of challengers, we introduce a bellicose diffusion strategy: When an individual makes one step random walk on the square lattice, it always moves to a site occupied by some one, and when more than two sites are occupied, it always challenges the strongest among them. An individual is prohibited to fight suscessively with the same opponent. Employing the same rule for the fighting and relaxation processes as Bonabeau et al[1], we examined the emergence of hierarchy and spacial structure in this society by MC simulation.

\section{Monte Carlo simulation}

MC simulation was performed for $N=3500$ individuals on the $L \times L$ square lattice with periodic boundary conditions from $L=60$ to $L=600$. 
Figure 1 shows the dependence of the order parameter on the population density. We see the transition occurs at $\rho \simeq 0.04$ when $\mu=0.1$ and $\eta=0.05$, which is much lower than the critical value for no-preference society ( $\rho \simeq 0.1$ for the same $\mu$ and $\eta$ ) studied by Bonabeau et al[1].

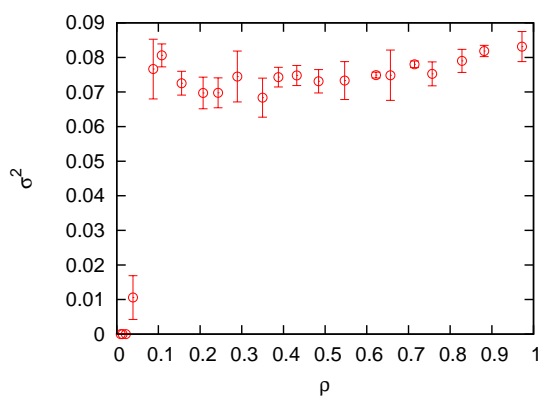

Figure 1: Order parameter $\sigma^{2}$ as a function of $\rho=N / L^{2}$ for $\mu=0.1$ and $\eta=0.05$.

The detailed structure in population is monitored by the profile of the winning frequency $W_{i} / X_{i}$. Figure 2 shows the profile of the winning frequency for four different population density; $\rho=0.022,0.056,0.086$ and 0.714 . In the egalitarian society at low densities below the critical density, the profile shows a sharp peak at $W_{i} / X_{i}=0.5$. When the density exceeds the critical value, the distribution of the winning probability becomes widespread, and at the same time individuals with winning probability above 95\% and with winning probability less than 5\% emerge,

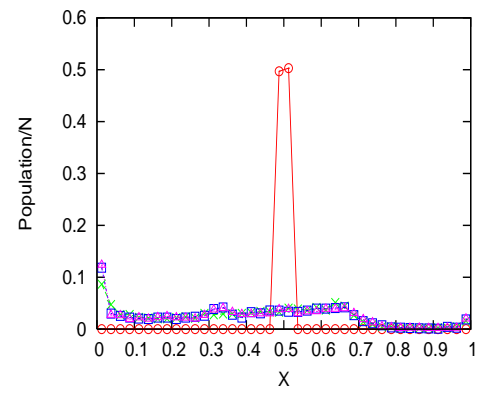

Figure 2: The profile of the winning frequency for four different densities $\rho=0.022(\bigcirc)$, $0.056(\times), 0.086(\square)$ and $0.714(\triangle) .(\mu=0.1$ and $\eta=0.05$. $)$

We conventionally classify individuals into three groups by the number of fights which an individual won; winners are individuals who won more than $2 / 3$ of fights 


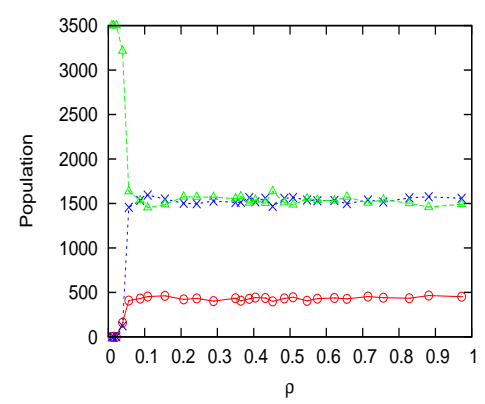

Figure 3: Dependence of the population in each class on the density when $\mu=0.1$ and $\eta=0.05$. Winners $(\bigcirc)$, losers $(\times)$ and middle class $(\triangle)$.

and losers are individuals who won less than $1 / 3$ of fights. Individuals between these two groups are called middle class. Figure 3 shows the population of each class as a function of the population density. It is interesting to note that the emergence of the hierarchy is signified by appearance of small number of winners. This is a clear contrast to a timid society where individuals always avoid fighting [2]. In the timid society, the hierarchical society emerges in two steps; the first and the second transition are signified by appearance of losers and winners, respectively.

We now proceed to examine the spatial structure of each state in the steady state, which is shown in Fig. 4. In the egalitarian society, no spatial structure is observed. When the population density exceeds the critical value, villages emerge, each of which consists of small number of winners and large number of middle class and losers. The size of the largest village depends strongly on the density; At the density just above the critical value, all individuals belong to one compact cluster as shown in Fig. 4(b). As the density is increased, the number of clusters increases and thus the size of the largest cluster is rather small (Fig. 4 (c)). When the density is larger than a critical percolation density, one large cluster appears which percolates the system (Fig. 4(d)). The critical percolation density is about 0.65 , which is larger than the critical percolation density 0.593 of the square lattice. This is due to the fact that in the model under consideration individuals have effectively strong attractive interaction [5].

We see that winners (red dots) are near the center of the village, surrounded by people in the middle class (green dots), and losers (blue dots) are at its perimeter. For $\rho=0.086$, we compare the population profile of winning frequency of each village, which 


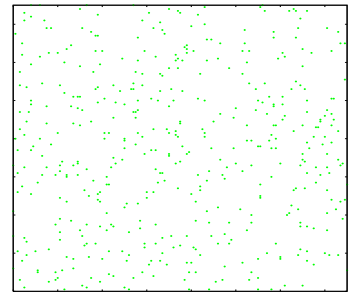

(a)

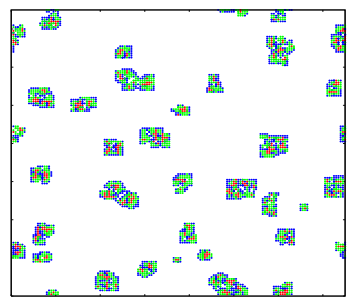

(c)

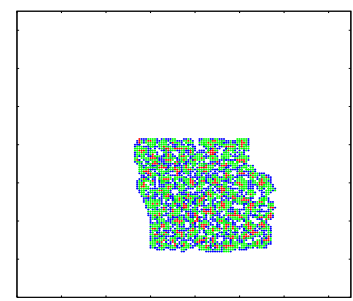

(b)

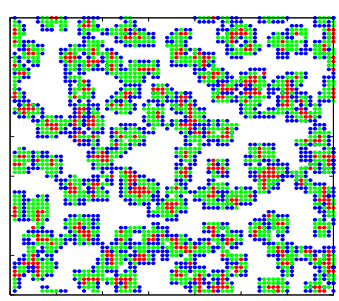

(d)

Figure 4: Self-organization of villages in the challenging society when $\mu=0.1$ and $\eta=0.05$. (a) No villages appear at $\rho=0.022$., (b) One big village is formed at $\rho=0.056$. (c) Many villages appear at $\rho=0.086$. (d) Villages form a percolating cluster at $\rho=0.714$. Winners, losers and middle class are represented by red, blue and green dots, respectively.

is shown in Fig. 5 It is interesting to observe that the profile is more or less common for all villages. This may be compared with the structure of medieval villages, where a few people dominate the village with many subordinates. The number of villages observed in the observation time depends on the population density. At higher densites, villages form a percolating cluster, corresponding to the borderless situation.

\section{Discussion}

We have shown that in a bellicose society the hierarchy self-organizes at much lower population density compared with the no-preference or a pacifist societies. Among the basic processes of diffusion, fighting and relaxation, a small change in the diffusion process affects significantly the self-organiztion of the social structure. In particluar, preference in the diffusion process plays an important role in the formation of spatial structure. The reason for the villages to be formed in the bellicose society is in the 


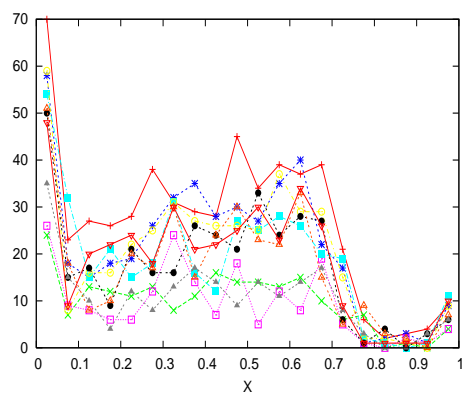

Figure 5: Population profile of winning frequency of each village at $\rho=0,086 . \mu=0.1$ and $\eta=0.05$.

effective attraction between individuals due to the diffusion algorithm, namely an individual always stay in the visinity of other individulas. Therefore the formation of villages is somewhat similar to the condensation of droplets in a gas.

In this paper, we have discussed the emergence of villages in the time period of our $\mathrm{MC}$ simulation. It is an open question to find out the distribution of villages in the long time limit. In fact, there are no mechnism to keep the center of mass of each village at the same position and thus each village can diffuse and may collide and merge with other village.

Another open and important quesition is to see the effect of the range of the random walk. The distance of one step of the random walk represents the mode of transportation. Therefore, as the mode of transportation advances, the effective population density is considered to increase and thus the globalization may occur at lower population density. These questions will be studied in the future. One can expect that various structures of society can be analyzed within the same frame work, which will eventually help in proposing the right policy.

\section{References}

[1] E. Bonabeau, G. Theraulaz, J.-L. Deneubourg, Physica A 217 (1995) 373.

[2] T.Odagaki, M. Tsujiguchi, Physica A 367 (2006) 435.

[3] A.O. Sousa, D. Stauffer, Int. J. Mod. Phys. C 11 (2000) 1063. 
[4] D. Stauffer, Int. J. Mod. Phys. C 14 (2003) 237.

[5] J.L. Duckers, R.G. Ross, Phys. Lett. 49 A (1974) 361. 